\title{
Actualidades en la sobrevida del carcinoma hepatocelular
}

\author{
Update in survival hepatocellular carcinoma
}

\author{
Eduardo E. Montalvo-Javé1,2*, Ana E. Hoyos-Lazaro', Mariana Espejel-Deloiza',
} Jonathan Chernitzky-Camaño ${ }^{1}$, Alejandro Rodríguez-Báez ${ }^{2}$ y Luis H. Ortega-León ${ }^{2}$

${ }^{1}$ Departamento de Cirugía, Facultad de Medicina, Universidad Nacional Autónoma de México; ${ }^{2}$ Servicio de Cirugía General, Clínica de Cirugía Hepato-Pancreato-Biliar, Hospital General de México. Ciudad de México, México

\section{Resumen}

Objetivo: Realizar una revisión de la literatura actualizada, abordando los aspectos clínicos y epidemiológicos más relevāntes asociados con la sobrevida del carcinoma hepatocelular y su relación con las estrategias terapéuticas que han demostrado un mejor resultado. Método: Se realizó una búsqueda bibliográfica empleando las bases de datos electrónicas Medline, Embáase, Springerlink y PubMed para identificar documentos publicados desde el 1 de enero de 1985 hasta agosto de 2020. Resultados: Se analizaron el abordaje y el manejo quirúrgico del carcinoma hepatocelular en todo el mundo, comparando las dos principales estrategias terapéuticas que reportan mayor porcentaje de sobrevida global. Conclusiones: En el protocolo de estudie del carcinoma hepatocelular es necesario un enfoque multidisciplinario. Los pacientes con carcinoma hepatocelular avanzado se beneficiarían de una estrategia multimodal individualizada que consista en resección y en otros casos con el trasplante hepático.

Palabras clave: Cáncer primario de hígado. Carcinoma hepatocelular. Sobrevida. Supervivencia acumulada.

\begin{abstract}
Objective: To offer an update of the literature, including clinical and epidemiological aspects associated with the survival of hepatocellular carcinoma. Method: A literature search was conducted using the electronic databases Medline, Embase, Springerlink and PubMed to identify papers published from January 1, 1985 to August 2020. Results: This study analyzed the sürgical approach and management of hepatocellular carcinoma worldwide, comparing the two main therapeutic strategiesthat report the highest percentage of global survival. Conclusions: According to the protocol of study, a multidisciplinary approach is necessary. Patients with advanced hepatocellular carcinoma would benefit from an individualized multimodal strategy consisting of hepatic resection and, in other cases, liver transplantation.
\end{abstract}

Key words: Primary liver cancer. Hepatocellular carcinoma. Survival. Accumulated survival.

\section{Introducción}

En todo el mundo, el carcinoma hepatocelular $(\mathrm{CHC})$ es la sexta neoplasia más frecuentemente diagnosticada y la cuarta causa de muerte asociáda a neoplasias reportada en 2018. El cáncer de hígădo ocupa globalmente el séptimo lugar en incidenciă, y se reporta como la quinta neoplasia más frecuentê en

Correspondencia:

*Eduardo E. Montalvo-Javé

Avda. Dr. Balmis, 148

Col. Doctores, Del. Cuauhtémoc

Fecha de recepción: 17-06-2019

C.P. 06720, Ciudad de México, México

E-mail: montalvoeduardo@hotmail.com

0009-7411/0 2019 Academia Mexican (http://creativecommons.org/licenses/by-nc-nd/4.0/).
Fecha de aceptación: 25-11-2019

DOI: $10.24875 / C I R U .19001375$
Cir Cir. 2021;89(1):10눈 114

Contents available at Pub̆Med

www.cirugiaycirujanos.com 
el sexo masculino y la novena en el sexo femenino. Se estiman mundialmente un total de 841,000 casos nuevos y 782,000 defunciones anuales. Los datos recientes indican que continúa incrementándose a diario en todo el mundo, con un predominio geográfico en Asia y África, representando el $85 \%$ de los casos reportados. La edad de presentación varía, con tendencia a aumentar de manera progresiva en todas las poblaciones, con predominio por el sexo masculino durante la quinta y la sexta décadas de la vida ${ }^{1,2}$. La distribución del CHC varía ampliamente según la localización geográfica, el grupo étnico y las distintas regiones en un mismo país (Fig. 1). Las zonas que registran mayor incidencia reportan más de 15 casos por cada 100,000 habitantes y año, cifra que corresponde al $40 \%$ del total de los casos reportados; por ejemplo, en la República Popular China, cada año se registran 137,000 casos $^{3,4}$. En las zonas con una baja incidencia se presentan apenas tres casos por cada 100,000 habitantes. En los Estados Unidos de América ha aumentado significativamente durante las últimas dos décadas, en especial en el grupo de edad de 45-49 años ${ }^{5-7}$. Con respecto al comportamiento de esta patología en México, en los últimos 30 años se han observado incrementos en la incidencia y la mortalidad del CHC; sin embargo, es necesario considerar la posibilidad de un subregistro de casos. El grupo investigador de Cortés-Espinosa, et al. ${ }^{8}$ demostró la duplicación de dicha incidencia en un período de 25 años comprendido entre 1965 y 1990, mediante un estudio retrospectivo en un hospital de la Ciudad México. Datos más recientes, registrados y analizados por Méndez-Sánchez, et al. ${ }^{9}$, concluyen que hubo un incremento nacional en la mortalidad por $\mathrm{CHC}$ del $14 \%$ como causa específica de muerte, pasando de 4.16 a 4.74 defunciones por cada 100,000 habitantes durante 6 años. Este incremento en la mortalidad ha alcanzado un $31 \%$ en el mismo periodo. Además, se encontró que el sexo femenino reportó la mayor mortalidad, con un incremento del $15 \%$, frente al $12.5 \%$ en el sexo masculino, en comparación con la población mundial. Con respecto a la edad, la mortalidad más alta se registró en pacientes mayores de 60 años. Los datos del Sistema Nacional de Información en Salud (SINAIS) corroboraron este incremento, con un aumento del $0.4 \%$ en la década de 1980 al $1.3 \%$ en el año 2008.

El índice de supervivencia, en todo el mundo, aún se mantiene muy bajo debido a las características biológicas y genéticas del tumor, aunado a la resistencia que manifiesta frente al tratamiento neoadyuvante, principalmente a los agentes quimioterapéuticos, y la pobre respuesta a la radioterapia.

Con el objetivo de impactar significativamente en la sobrevida de estos pacientes se ha propuesto como tratamiento de primera línea la cirugía; sin embařgo, los expertos continúan desarrollando nuevos y combinados abordajes que permitan individualizar eletratamiento que conceda un mejor pronóstico a corto y largo plazo.

En la actualidad, la resección hepática se considera el tratamiento de primera línea para aquellos pacientes que presentan tumores únicos, con un tamaño $\leq 3 \mathrm{~cm}$ y con una función hepática conservada j, no obstante, se encuentra limitada porque la mayoría de los pacientes, incluso los casos que reportan tumores pequeños, han desarrollado falla hepática prequirứrgica debido al diagnóstico tardío en fase avanzadã de la enfermedad o posquirúrgica, afectando de manera significativa la sobrevida a corto plazo y con un álto porcentaje de recurrencia del cáncer $(50 \%)^{10}$. El trasplante de hígado, por otro lado, se considera unğpotencial tratamiento "curativo», puesto que resecă el cáncer infiltrado en conjunto con el parénquima hẹpático dañado ${ }^{11,12}$. Los pacientes candidatos a esta cirugía hepática que cumplen los criterios de Mitán, inicialmente descritos por Mazzaferro, et al. ${ }^{13}$, han sido aceptados y validados por el panel de expertos dè la Conferencia de Barcelona 2000 y la clasificacióñdel Barcelona Clinic Liver Cancer Group, como estrictos criterios de selección para la estadificación del arcinoma hepatocelular; dichos criterios se asocian con una sobrevida a 4 años del $75 \%$. El mejor abordaje es sin duda el quirúrgico, hablando del trasplante dẻ hígado y la resección hepática, ambos agresivos, embargo, con mejores resultados registrados: el $\_$primero debido al pronóstico y porcentaje de sobrevida a corto y largo plazo con respecto a la resección quirúrgica y ésta por la ventaja que brinda al "ganar tiempo» en caso de no tener un donante disponible $e^{\overline{1}+, 15}$. Se modifica la referencia por un artículo que concentra artículos de revisión y análisis multicéntricos e i i for rmes resumidos de consensos recientes que fortaiece el argumento. El propósito de esta revisión se bašará únicamente en la comparación del trasplante hepấtico versus resección hepática por ser la mejor estratęgia combinada para tratar el cáncer primaria de hígado.

\section{Método}

Se realizó una búsqueda bibliográfica empleañdo las bases de datos electrónicas Medline, Embase, 


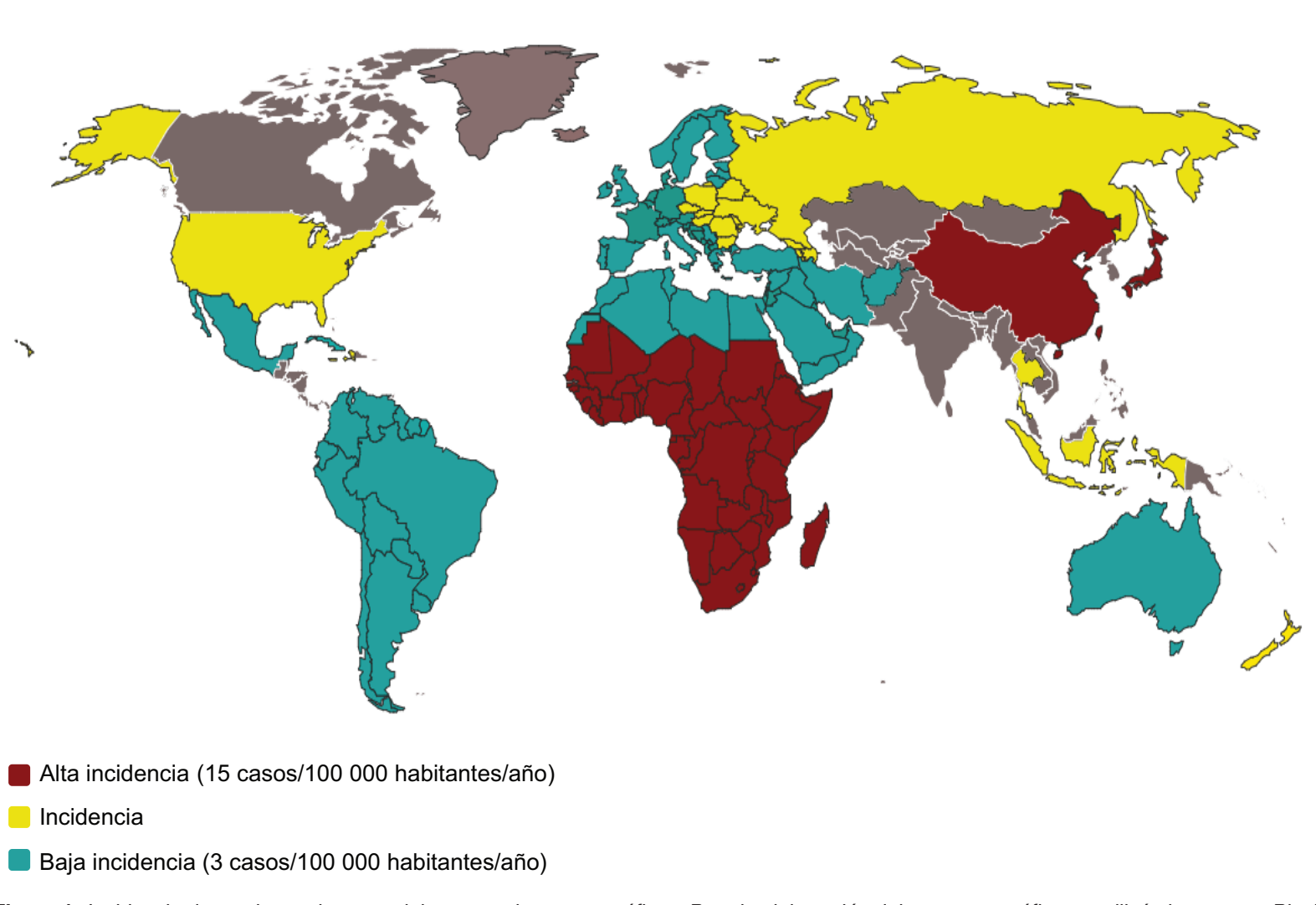

Figura 1. Incidencia de carcinoma hepatocelular por regiones geográficas. Para la elaboración del mapa geográfico se utilizó el programaßBixel Map Generator (https://pixelmap.amcharts.com/).

Springerlink y PubMed para identificar documentos publicados desde enero de 1985 hasta agosto de 2020, utilizando los siguientes términos MeSH: [hepatocarcinoma cellular] [primary liver cancer] [epidemiology] [diagnostic] [treatment] [surgical treatment] [survival] y [follow up]. Se seleccionaron 53 referencia. Los criterios de inclusión fueron: publicaciones a partir del 1 de enero de 1985 y hasta agosto de 2020 relacionadas con la sobrevida del $\mathrm{CHC}$, la fisiopatología y la epidemiología mundial disponibles en revistas indexadas a través de la biblioteca médica virtual de la Facultad de Medicina de la Universidad Nacional Autónoma de México UNAM en inglés y español. Las publicaciones que no fueron seleccionadas se excluyeron por no cumplir con los criterios anteriores o bien porque no reportaban datos de interés sobre el tema.

\section{Factores de riesgo}

Se conocen muchos factores de riesgo que se encuentran estrechamente relacionados con el aumento de la incidencia (Tabla 1) y de la mortalidad del CHC (Fig. 1).

\section{Grupo étnico}

Con respecto al grupo étnico, en un estudio realizado en 2014 en los Estados Unidos de Américaz̄opor Setiawan, et al. $^{16}$, en el que se investigaban los factores de riesgo para cáncer de hígado y otras enfermedades crónicas hepáticas, se identificó una mayor tasa de incidencia en grupos étnicos específicos, como latinos $(22.5 / 100,000)$, hawaianos nativos (21.3/100,000), japoneses americanos $(16.8 / 100,000)$ y afroamericanos $(16.6 / 100,000)$, frente a los cauccásicos $(7.5 / 100,000)$, mostrando una marcada diferencia con los primeros.

\section{Edad}

La edad de presentación varía según la localización geográfica, con una tendencia a aumentar progresivamente en todas las poblaciones, con predominió en el sexo masculino durante la quinta y la sexta dẹćcadas de la vida. La edad media de presentación en Europa Occidental, Asia y México oscila alrededơ⿱ de los 60 años, mientras que en África Subsahariana hay 
Tabla 1. Factores de riesgo identificados asociados a carcinoma hepatocelular

Edad: quinta y sexta décadas de la vida (> 70 años)
Sexo masculino
Etnia
Cirrosis hepática
Infección crónica por VHB
Infección crónica por VHC
Hemocromatosis
Alcoholismo
Intoxicación por aflatoxina B1
Radiación ionizante
Glucemia elevada/diabetes mellitus tipo 2
Síndrome metabólico/obesidad
Hipotiroidismo
Hígado graso
Esteatohepatitis no alcohólica
Anomalías genéticas: deficiencia de alfa-1 antitripsina, tirosinemia,
galactosemia y fructosita
Predisposición genética
Hormonas anabolizantes
Anticonceptivos estrogénicos
Enfermedad de Wilson en presencia de cirrosis

una marcada disminución de la edad hasta una media de 33 años ${ }^{17}$.

\section{Toxinas alimenticias}

La intoxicación con aflatoxina B1 se relaciona con un inadecuado almacenamiento de cereales, y algunas comidas que activan un efecto sinérgico con patologías subyacentes del hígado de carácter inflamatorio cróni$\mathrm{co}$, como la cirrosis y la fibrosis, frecuentemente asociadas a infecciones de etiología viral, o bien por alcoholismo crónico, que confieren un microambiente adecuado para desarrollarse, a través de una falta de regulación genética en el mecanismo cíclico caracterizado por la necrosis y la regeneración del hepatocito.

\section{Sexo}

Con respecto al sexo, las regiones que reportan mayor incidencia indican que la relación hombre:mujer es de 5.7:1. La proporción disminuye a 2.4:1 en las zonas con una incidencia intermedia y baja. Por ahorā no hay una explicación satisfactoria para este fenómenno. Se ha especulado acerca del papel que tienen los estrógenos y los andrógenos como moduladores de la hepatocarcinogénesis ${ }^{18}$.

\section{Infección por los virus de las hepatitis B y C}

Se reconoce como principal factor de riesgo lă infección por el virus de la hepatitis B (VHB) en áreas con una mala cobertura de vacunación y por el virus de la hepatitis C (VHC) en los países subdesarrollados. Las áreas geográficas con mayor incidencia presentan este patrón infeccioso, casi endémico, quê se asocia a enfermedad hepática crónica y el consecuente desarrollo de CHC. En América y Europa la prevalencia es mucho menor debido a las políticas vacunales. Por ese motivo, la asociación entre lahepatopatía viral crónica y el $\mathrm{CHC}$ dirige las estrategias preventivas que incluyen la aplicación universal de la vacuna contra la hepatitis $B$ al nacer y los programas destinados a detener la transmisión y la erradicación temprana de las hepatitis $B$ y $C^{19,20}$.

\section{Fisiopatología}

El conocimiento de los mecanismos moleculares implicados en la biología de la hepatocarcinogénesis aún no es completo. Las diferentes señalizaciones bioquímicas y genéticas que dan origen al $\mathrm{CHC}$ iñvolucran inicialmente un microambiente hepático modificado relacionado con una inflamación crónicadel hígado que se acompaña de fibrosis masiva, ambas causantes de una acumulación de alteraciones gènéticas en las células hepáticas normales. Los hepatocitos sufren una modificación estructural de carácter displásico que da lugar al carcinoma temprano đue progresa a $\mathrm{CHC}$. Este cáncer se caracteriza por ser heterogéneo y sólido ${ }^{21}$. La intensa actividad angiogénica en el hígado durante la infección por el WHC contribuye al alto riesgo que tienen estos pacientes para desarrollar $\mathrm{CHC}$, en comparación con la infección por el VHB, que se relaciona con un estado inflamatorio crónico que genera estrés oxidativo mediado por dos vías: una dependiente de proteḷnas transformadoras HBVx o NS5, y otra dependiente de proteínas core capaces de modificar al hepatocito 22,23 . Desde el punto de vista molecular, el $\mathrm{CHC}$ esiūna enfermedad compleja que muestra heterogeneidad 
entre los diferentes nódulos tumorales que se forman en un mismo paciente.

\section{Aspectos clínicos}

En su fase inicial suele tener una presentación clínica asintomática, que puede permanecer así durante meses e incluso años; esto significa que el $\mathrm{CHC}$ debe contemplarse siempre dentro del diagnóstico diferencial de todas las patologías crónicas del hígado, aun cuando su aparición no se encuentre ligada al antecedente de infección viral del hígado ni a cirrosis.

Cuando es sintomático hay diferentes formas de presentación clínica; por ejemplo, en África Subsahariana, la forma más frecuente de presentación clínica es la aparición de lesiones de gran tamaño, palpables a la exploración física, que ocupan el cuadrante superior derecho del abdomen, con compromiso del parénquima hepático que suele cursar sin evidencia de enfermedad extrahepática a pesar de sus dimensiones. Por otro lado, se ha descrito una presentación aguda que cursa con hemoperitoneo secundario al estallido de las lesiones. En los pacientes con una patología inflamatoria subyacente, como cirrosis, el $\mathrm{CHC}$ cursa con ascitis, encefalopatía, ictericia o sangrado de origen varicoso ${ }^{24}$. Los pacientes sin antecedentes de infección viral o cirrosis cursan en general con una forma grave de la enfermedad, de rápida evolución y con una alta tasa de mortalidad. La mayoría de estos pacientes presentan al momento del diagnóstico una fase avanzada del cáncer con compromiso intrahepático y extrahepático que contraindica por completo el abordaje quirúrgico.

\section{Estadificación}

No existe un consenso universal sobre cuál de todos los sistemas de estadificación es el mejor para clasificar esta patología. Actualmente, el sistema de estadificación de la Clínica del Cáncer de Barcelona (BCLC, Barcelona Clinic Liver Cancer) es el más utilizado debido a los criterios que toma en cuenta para la evaluación del paciente, las características del tumor y la función hepática. El sistema clasifica a los pacientes en etapa muy temprana, etapa temprana, etapa intermedia, etapa avanzada y etapa terminal, de acuerdo con el tamaño del tumor, la invasión vascular, el número de nódulos tumorales y la presencia de metástasis. Este sistema ofrece un algoritmo para el manejo del paciente según la etapa en que se clasifique. Los pacientes que se encuentran en etapa muy temprana o temprana son considerados candidatos para tratamiento curativo, que comprende la resección hepática, el trasplante hepático o la terapia de ablación local, que reduce en un porcentaje el tumor para posteriormente ser resecado. Los pacientes en etăpa intermedia, principalmente los que presentan múltiples nódulos con diámetros $>10 \mathrm{~cm}$, no son considerados candidatos para tratamiento quirúrgico, pero sí se consideran para un abordaje con potencial control de la actividad angiogénica y tumoral a través de la-quimioembolización transarterial, que busca reduci⿺ la mutación celular y el riesgo de sangrado. Para aquellos pacientes que se encuentren en etapa terminal demostrada, con invasión de la vena porta o metástasis distantes, se considera el uso de agentes quimioterapéuticos sistémicos, como sorafenib, o bien son reclutados para estudios clínicos que ofrecen fármacos y terapias combinadas experimentales. A pesar de su estándar de referencia, el sistema de clasificación BCLC continúa en controversia debido a sus limitaciones en escenarios excepcionales ${ }^{25,26}$.

\section{Auxiliares diagnósticos}

El uso de diferentes técnicas de imagen, comōultrasonido, tomografía computada y resonancia magnética, tiene como objetivo ayudar a confirmar el diagnóstico, mientras que la histopatología es considerada el método de referencia para el diagnóstico.

\section{Ultrasonografía}

El ultrasonido se considera el primer métod $\bar{\sigma}$ de tamizaje para la detección y la evaluación de nódulos malignos en pacientes con seguimiento de una hepatopatía crónica o con una condición de riesgo, ya que es accesible económicamente y se encuentra dişponible en casi cualquier parte del mundo. La efectividad del ultrasonido para detectar cualquier formā de $\mathrm{CHC}$ es elevada (94\%), pero la sensibilidad disminuye cuando la lesión diana es muy pequeña $(63 \%)$ EI ultrasonido establece un intervalo de 6 meses como periodo de vigilancia del proceso de desarrollo y ềvolución de las lesiones.

A pesar de las estrategias de prevención y detección temprana que se ha buscado implementar, ưna vez establecido el diagnóstico la sobrevida a 5 años es menor del $10 \%$, en parte debido a la resistencia que existe a los agentes quimioterapéuticos y alülevado porcentaje de recurrencia de este cáncer ${ }^{27}$. 
La sensibilidad y la especificidad de los estudios radiológicos convencionales ofrecen un uso diagnóstico y terapéutico que permite dar directriz al tratamiento ${ }^{28}$.

\section{Sobrevida}

El promedio de sobrevida del $\mathrm{CHC}$ está relacionado con los factores de riesgo del paciente, las características del tumor, su estadificación y la función hepática, es decir, si se encuentra conservada o hay insuficiencia o falla hepática clínicamente demostrable. Un abordaje inicial oportuno que se acompañe de un estadio temprano del carcinoma es el escenario ideal asociado con un mejor pronóstico. Sin embargo, como hemos visto, la mayoría de los pacientes, una vez realizado el diagnóstico, se enfrentan a un estadio avanzado de la enfermedad que reduce significativamente el porcentaje de sobrevida a corto y muy corto plazo $^{29}$. Si analizamos los abordajes terapéuticos, las dos modalidades de intervención quirúrgica, resección hepática y trasplante de hígado, son hasta el momento las que han demostrado ser las mejores alternativas en casi cualquiera de las etapas de estadificación, brindando al paciente una sobrevida media de hasta 44.7 meses, en comparación con la quimioembolización transarterial, que ofrece una sobrevida media de apenas 23.7 meses $^{30}$. Se han descrito factores de mal pronóstico que incrementan el riesgo de mortalidad en las poblaciones y que por supuesto impactan directamente en la sobrevida a corto y largo plazo (Tabla 2).

\section{Resección hepática}

La resección hepática se reporta como una de las dos estrategias terapéuticas con mayor beneficio para el paciente en una etapa clínica inicial. Los análisis comparativos durante las últimas décadas (Fig. 2) demuestran el buen pronóstico que hay a corto y mediano plazo ${ }^{31-34}$. En 2014 se reportó un grupo de pacientes con $\mathrm{CHC}$ avanzado y función hepática conservada, en los que la resección hepática alcanzó un porcentaje de supervivencia acumulada a 1,3 y 5 años de seguimiento del $88 \%$, el 62 y el $39 \%$, respectivamente ${ }^{33}$. En otro estudio retrospectivo realizado en 2016 se analizaron las asociaciones que hay entre el sistema de estadificación BCLC y la sobrevida promedio a 5 años posterior a la resección hepática en tumores que no cumplen con los criterios de Milán, la cual corresponde a un $72.4 \%$ para el estadio 0 , un \begin{tabular}{l} 
Tabla 2. Factores relacionados con un peor pronóstico y elevada \\
mortalidad asociada a carcinoma hepatocelular \\
\hline Edad $\geq 60$ años
\end{tabular}

$66.3 \%$ para el estadio $A$, un $36.9 \%$ para el estadio $B$ y un $28.9 \%$ para el estadio C; esto representa unpanorama alentador para aquellos pacientes que seen un tumor único o múltiples nódulos de ṃ̂y grandes dimensiones que tienen oportunidad desometerse a un abordaje quirúrgico como tratamiēnto potencialmente curativo ${ }^{35,36}$. El riesgo de recurreñcia se explica por la posibilidad de desarrollar metástäsis intrahepáticas o la aparición de novo de CHC ençpacientes con tejido cirrótico remanente; afortunadamente, estos pacientes continúan siendo candidătos para trasplante y pueden someterse a quimioembolización transarterial durante el tiempo de esperasdel trasplante. En los pacientes con un $\mathrm{CHC}$ recurrente con invasión microvascular demostrada, el tratamiento quirúrgico continúa recomendándose como deçorimera línea puesto que una segunda resección hepática ofrece una sobrevida promedio de 49 meses, y sin invasión microvascular se extiende hầsta 61 meses, en comparación con aquellos pacientes que no se sometían a una segunda resección hèpática y únicamente eran tratados con quimioembolización transarterial, reportando una sobrevida de apenas 32 meses posterior a una recurrencia ${ }^{37} \mathrm{El}$ promedio de sobrevida de la resección hepáticâ se encuentra condicionado por el «volumen remanente mínimo hepático», un término que hace referencâa al porcentaje mínimo aceptado para realizar este äbordaje y tener un mejor resultado a largo plazo. Generalmente este porcentaje corresponde a un $40 \%$ del total del parénquima, pero en la mayoría de los cientes representa un factor de mal pronóstico debido a que dicho remanente tiene un componente inflamatorio cirrótico que favorece la recurrencia de ésta enfermedad.

Algunos autores proponen nuevas técnicas quirúrgicas basadas en una subsegmentación anatómica que permite realizar la resección hepática de forma 


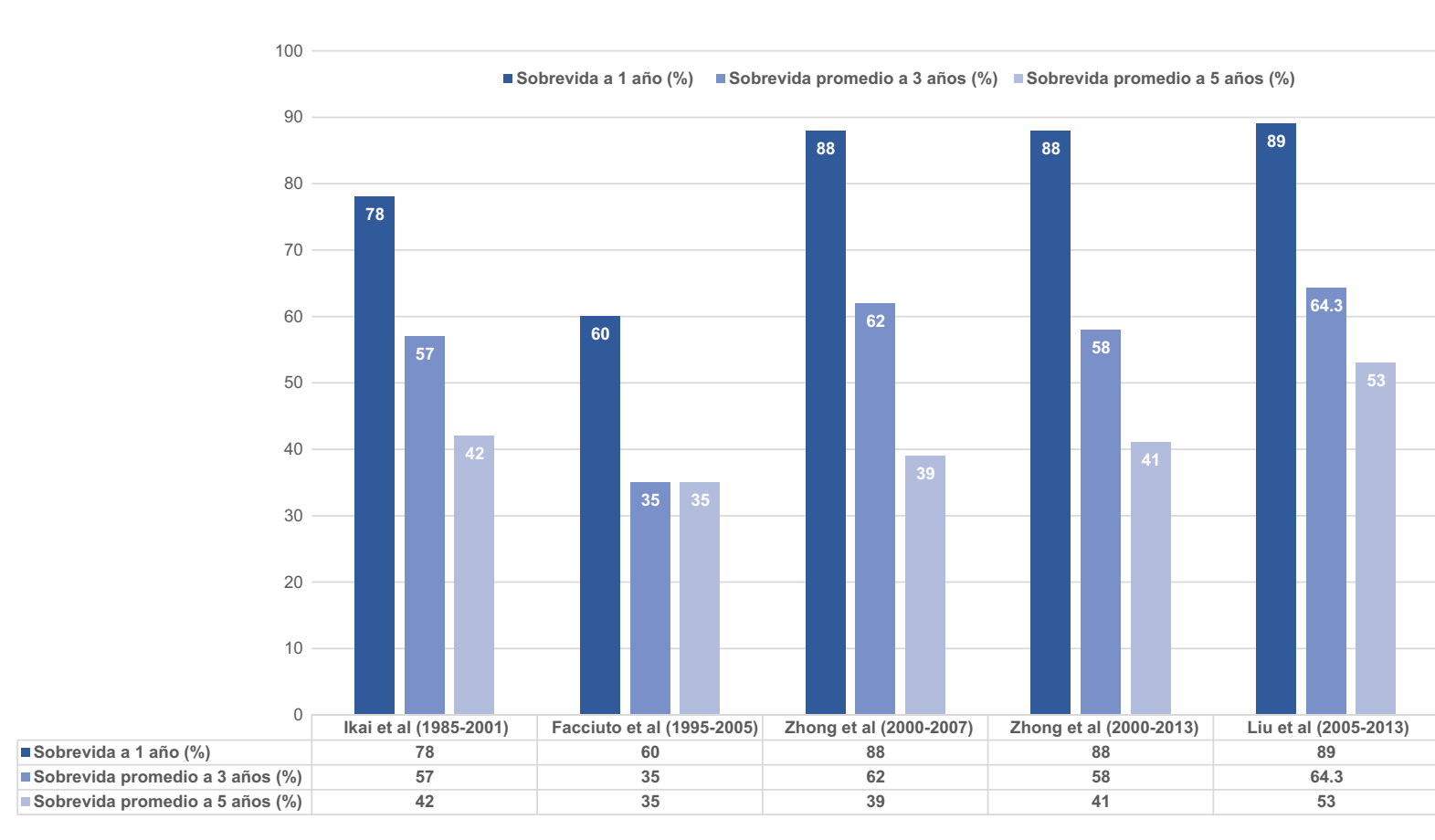

Figura 2. Análisis comparativo de sobrevida global por década en pacientes con carcinoma hepatocelular a quienes se realizó resêeción hepática.

más organizada. Esta técnica ha demostrado tener la capacidad de disminuir el riesgo de metástasis intrahepáticas a través de la vena porta en comparación con la hepatectomía menor no anatómica en pacientes con $\mathrm{CHC}$ de dimensiones entre 2 y $5 \mathrm{~cm}$ de diámetro. En casos con neoplasias más pequeñas se acepta la hepatectomía menor no anatómica puesto que el riesgo de diseminación portal es mínimo ${ }^{38}$.

\section{Trasplante de hígado}

El trasplante hepático ortotópico es considerado el tratamiento de elección para pacientes con una clasificación Child-Turcotte-Pugh de clase B o C, cirrosis o diagnóstico de $\mathrm{CHC}$ en estadio inicial ${ }^{39}$. En pacientes con una función hepática conservada y un $\mathrm{CHC}$ en estadio temprano ofrece una sobrevida del $87 \%$ a 1 año, del $69 \%$ a 3 años y del $60 \%$ a 5 años. La recurrencia del $\mathrm{CHC}$ después de una resección hepática es frecuente, por lo que se propone reservar como siguiente paso en el algoritmo para su tratamiento al trasplante de hígado "de rescate», pues ofrece una segunda oportunidad en estos $\operatorname{casos}^{40}$.

Se reconoce que una proporción significativa excede los criterios de Milán, pero diferentes autores han reportado un porcentaje de sobrevida superior al $70 \%$ a 5 años de seguimiento en pacientes que exceden dichos criterios, por lo que se ha buscado ampliầlos y hacerlos más flexibles en pacientes con tumores más grandes o que hayan sido reducidos por trătamientos neoadyuvantes como la quimioembolización transarterial o la terapia de ablación por radiofrectencia local ${ }^{41}$.

A partir de este momento podemos observar que el tratamiento de un paciente con $\mathrm{CHC}$ exige ser individualizado de manera estricta y requiere ser sometido a una vigilancia estrecha por el alto riesgo de rêcurrencia que existe con este tipo de cáncer. Së ha propuesto que la indicación de trasplante de hígáado por $\mathrm{CHC}$ pueda ampliarse para incluir cualquier tumor solitario $<6.5 \mathrm{~cm}$, tres o menos nódulos en una lesión más grande que no rebase los $4.5 \mathrm{~cm}$ y con un diámetro total del tumor $<8 \mathrm{~cm}$. El análisis comparâtivo de los excelentes resultados obtenidos a lo large de las últimas décadas permite identificar el promediō de sobrevida global en el seguimiento de los pacientes con $\mathrm{CHC}$ que se sometieron a trasplante hepático, superando marcadamente a la resección hepática (Fig. 3). El trasplante hepático, sea de donante vivo o de cadáver, es la piedra angular en el tratamiểnto del $\mathrm{CHC}$, pues el promedio de sobrevida es mâyor comparado con la resección hepática y el porcentaje de recurrencia a 5 años es menor ${ }^{42-45}$. 


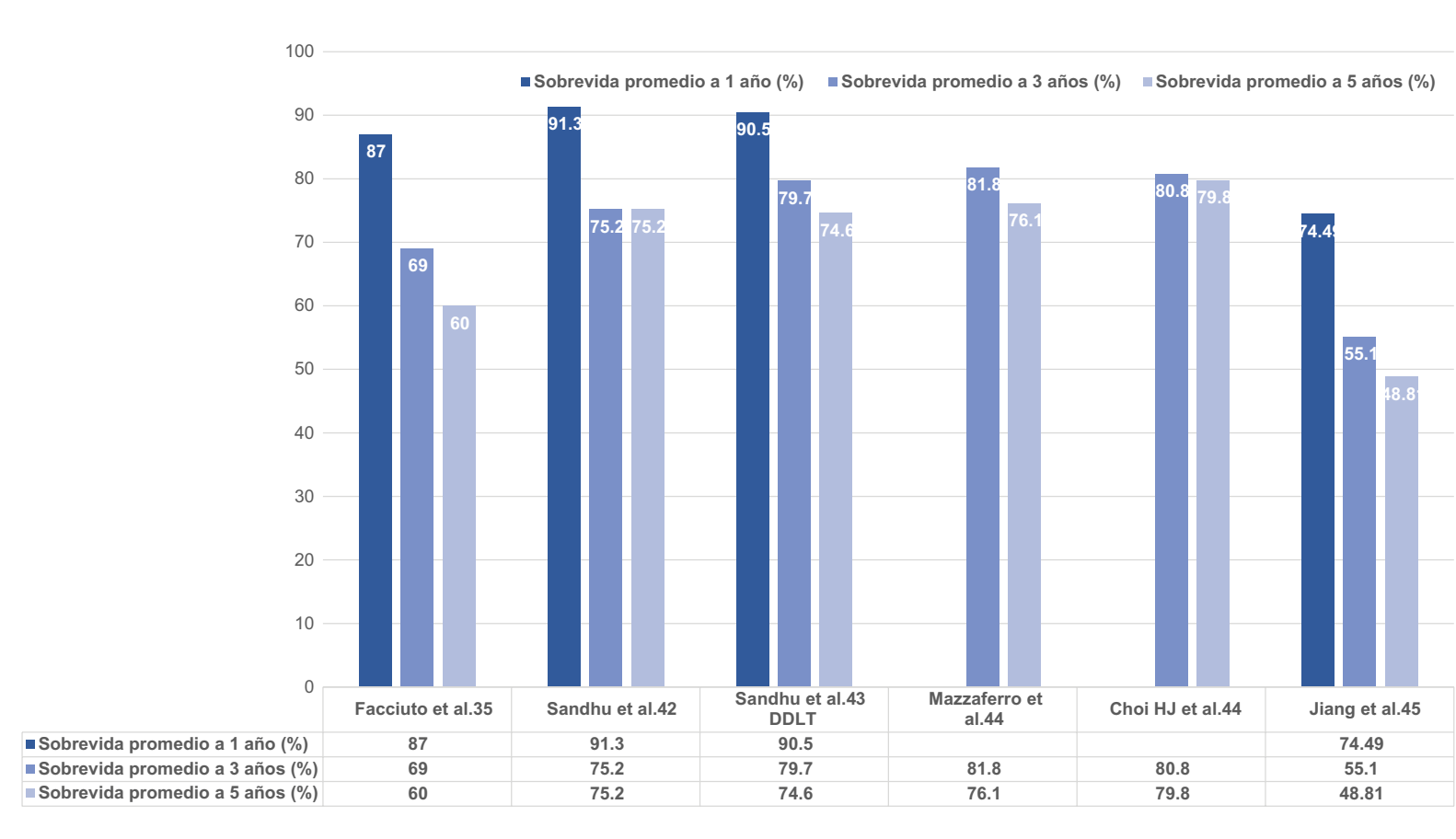

Figura 3. Análisis comparativo del promedio de sobrevida en el seguimiento de pacientes con carcinoma hepatocelular a quienes se rêalizó trasplante hepático.

\section{Nuevas propuestas terapéuticas}

Hay un interés clínico creciente en el uso de las estatinas para una serie de enfermedades crónicas, más allá de sus indicaciones tradicionales para la enfermedad cardiovascular. Recientemente se ha señalado el uso de estatinas, como la simvastatina, como tratamiento preventivo del $\mathrm{CHC}$. Las estatinas han mostrado propiedades antiinflamatorias, antifibróticas y regenerativas, lo que las convierte en una opción terapéutica interesante para las enfermedades hepáticas crónicas. Al principio existía una preocupación relacionada con la seguridad de este fármaco en pacientes con insuficiencia hepática, pero la evidencia que se tiene documentada ha proporcionado pruebas sólidas de que las estatinas son seguras y potencialmente benéficas en pacientes con afecciones catalogadas como precirróticas y cirróticas ${ }^{46}$.

Se han descrito hallazgos que sugieren que el uso de estatinas después de un diagnóstico de $\mathrm{CHC}$ se asocia con un menor riesgo de muerte para los pacientes. En 2019, un grupo de investigadores demostró que los pacientes con $\mathrm{CHC}$ que usaron estatinas después del diagnóstico del cáncer tenían un riesgo un $15 \%$ menor de cáncer específico y un riesgo un $11 \%$ menor de muerte en comparación con el grupo de pacientes con diagnóstico de $\mathrm{CHC}$ que no usaban estatinas. Se necesitan más estudios, con series de pacientes más grandes y criterios de valoración clínicos concretos, antes de que se pueda recomendar el uso de estatinas en los pacientes con enfermedad hepática crónica ${ }^{47}$.

Mundialmente se reporta un índice de supervivencia muy bajo, que se explica por la naturaleza biológica y genética del $\mathrm{CHC}$, y por la resistencia que existe àjos agentes quimioterapéuticos y la pobre respuesta la terapia adyuvante como la radioterapia. Con el objetivo de mejorar significativamente la sobrevida de estos pacientes se encontró que el tratamiento de primera línea corresponde a las intervenciones quirúrgicas agresivas que involucran la resección hepática, ăun cuando la estadificación clínica del tumor la limita en combinación con el trasplante de hígado, cuandō-se tiene disponible este recurso.

Se están desarrollando nuevas y diferentes estrategias para diagnosticar y tratar el $\mathrm{CHC}$ con el objêtivo de mejorar la sobrevida de los pacientes. Entre $=10 s$ métodos diagnósticos, el ultrasonido, por su sensibilidad y especificidad, continúa siendo de primera elección para la vigilancia, mientras que la histopatología, un método invasivo, se considera de referencia para el diagnóstico definitivo. Para el médico tratante representa un reto, pues en general el diagnóstico se hace tardíamente, lo que reduce de manera considerable la 
sobrevida global de los pacientes. Además, debido al alto porcentaje de regresión/reincidencia, se considera una patología difícilmente "curable», y de ahí que las diferentes estrategias terapéuticas que se han desarrollado en los últimos años tengan sus ventajas y desventajas. La resección hepática y la terapia de ablación mediante radiofrecuencia son consideradas tratamientos potencialmente curativos, pero existe recurrencia en aproximadamente el $50 \%$ de los casos dentro de los primeros 3 años posteriores a la resección y en más del $70 \%$ dentro de los primeros 5 años posteriores al procedimiento, posiblemente asociada a metástasis intrahepáticas. Los resultados de diversos análisis retrospectivos y metaanálisis revelan que el trasplante hepático, de acuerdo con los criterios de Milán, está asociado a una mayor sobrevida que la resección hepática. Lamentablemente, la disponibilidad de donadores es una gran limitante y en torno al $10 \%$ de los pacientes enlistados para este procedimiento mueren durante la espera. La hepatectomía parcial es el tratamiento óptimo para los pacientes con función hepática adecuada sin evidencia de hipertensión portal ni invasión vascular, y el trasplante hepático es potencialmente curativo para los pacientes en estadios tempranos, pero con inadecuada función hepática; sin embargo, la mayoría de los pacientes se presentan en estadios avanzados en los que ya no son candidatos para dicho tratamiento. De esta forma, se indica quimioembolización mediante cateterismo arterial y quimioterapia como procedimientos paliativos, con resultados desalentadores.

\section{Discusión}

El CHC es el tercer cáncer más agresivo en todo el mundo, en ambos sexos, después de los cánceres de pulmón y de colon ${ }^{48}$. Se han identificado muchos factores de riesgo estrechamente relacionados con el aumento de casos y de defunciones, entre los que destacan las infecciones por el VHB y el VHC, que dan lugar a diferentes y complejas interacciones celulares y moleculares que alteran el microambiente hepático; este fator de riesgo determina, en la mayoría de los pacientes, el pronóstico y la respuesta al tratamiento ${ }^{49}$.

La estadificación de la lesión tumoral cumple un papel decisivo en el abordaje terapéutico y en la toma de decisiones clínicas que convengan mejor a la sobrevida del paciente. Actualmente se encuentra disponible mucha evidencia que pone en controversia los estrictos criterios para someterse a una resección hepática o un trasplante hepático, y con frecuencia esto es motivo de discusión para el cirujano y su grupo multidisciplinaririo: qué hacer cuando un paciente no cumple con los criterios de Milán. Recordemos que se consideran únicas dos opciones con potencial curativo real gque registran menor tasa de recurrencia y de mortalidad ${ }^{50}$.

El tratamiento puede clasificarse en dos categorías principales: abordajes que son potencialmente curativos y abordajes que son paliativos. El tratamiênto quirúrgico continúa siendo el pilar de las opciones disponibles para su manejo, de las cuales la resección y el trasplante hepático lideran la lista, pero hay que tener en cuenta que la mejor opción de tratamiento siempre dependerá del paciente y de sus anteccedentes, específicamente de la función hepátică la presencia de cirrosis, la infección por VHB o VHC la hipertensión portal, el tamaño y la ubicación deEtumor, así como de su condiciones generales de salud. La decisión de realizar un trasplante hepático o una resección quirúrgica está determinada principalmênte por cuatro factores: la presencia de hipertensión §ŏ tal, el grado de cirrosis, la función hepática y la dilisponibilidad de un donante, asociado al riesgo de recurrencia postrasplante ${ }^{51}$.

Un nuevo enfoque para tratar a los pacientes con $\mathrm{CHC}$ consiste en usar más activamente una estrấtegia combinada de resección hepática y trasplante Es decir, en pacientes con una función hepática conservada y un $\mathrm{CHC}$ en etapa temprana, se ha deserito una estrategia que emplea la resección y el trasplante hepáticos de forma escalonada, y que ha mostrado buenos resultados que confieren al paciente unatmejor posibilidad de supervivencia ${ }^{52}$.

El problema que generan el abordaje y el manejo del $\mathrm{CHC}$ es motivo de discusión en todo el munido. En la actualidad, las líneas de investigación en relación con el CHC recomiendan seguir los lineamieǹtos más actuales reportados y al mismo tiempo individualizar el tratamiento, ajustándolo a las caracteristicas clínicas e histopatológicas de cada paciente ${ }^{53}$.

\section{Conclusiones}

La incidencia y la distribución del CHC varían ămpliamente dependiendo del grupo étnico, el sexo, la edad y sobre todo la distribución geográfica, pues predomina en el sureste de Asia y en África. La incidencia reportada en México es baja, pero descơnocemos si se debe a un subregistro de casos.

Los pacientes con factores de alto riesgo para $\mathrm{C}$ तC, como cirrosis, infección crónica por VHB o VHC, 
hepatopatía alcohólica y esteatohepatitis, con presencia de lesiones visibles por ultrasonido o tomografía contrastada trifásica, deben considerarse altamente sospechosos de CHC. La asociación que existe entre la prevención de la hepatopatía viral mediante la vacunación y el CHC explica por qué se considera una neoplasia prevenible.

Merece la pena hacer una mejora en la vigilancia epidemiológica de esta patología para conocer mejor su comportamiento y su distribución, pues la población mexicana cumple con varios de los factores de riesgo asociados al desarrollo de cáncer primario de hígado.

Antes de tomar una decisión terapéutica se debe evaluar la función hepática para identificar cuál es el abordaje que conviene mejor al paciente, así como el pronóstico inmediato, en el posquirúrgico, y a corto y largo plazo, para la sobrevida global del paciente. Para la evaluación clínica de la función hepática se pueden usar las escalas Child-Pugh y MELD (Model for End-stage Liver Disease), según las cuales el registro de puntuaciones bajas permite realizar una resección hepática, un trasplante ortotópico de donador vivo o de cadáver, o bien ofrecer al paciente una terapia neoadyuvante cuando las dimensiones del tumor impiden un abordaje quirúrgico.

Como ya hemos mencionado, la elección de un abordaje quirúrgico sobre otro dependerá principalmente de la presencia de hipertensión portal, de la función hepática y de la disponibilidad de un donante, pues el trasplante debe priorizarse por encima de todas las terapias neoadyuvantes disponibles e incluso de la resección hepática misma, debido al buen pronóstico que tiene a largo plazo. El trasplante hepático se considera como la solución al «matar dos pájaros de un tiro», ya que elimina el parénquima cirrótico y el tumor en su totalidad; sin embargo, el paciente debe cumplir con criterios específicos que eviten una descompensación posquirúrgica que ponga en peligro su vida. La resección hepática, en comparación, no tiene un promedio de sobrevida tan favorable debido al elevado porcentaje de reincidencia, por lo que puede considerarse una estrategia intermedia que busca "ganar tiempo» si no se cuenta con un donador inmediatamente, cuando el paciente se encuentra en lista de espera. Alternativamente, también se puede recurrir al uso de terapias adyuvantes como la quimioembolización transarterial o la terapia de ablación por radiofrecuencia para disminuir el tamaño del tumor y su actividad angiogénica. Uno de los mayores retos a los que se enfrenta un cirujano es la toma de decisiones con respecto al abordaje quirúrgico; por esta razón, el tratamiento se debe apegar a los lineamientos internacionalmente establecidos, pero al mismo tiempo debe ser individualizado y dirigido a lograr el mayor porcentaje de sobrevida a largo plazo.

\section{Financiamiento}

No se recibió ningún tipo de financiamiento para la elaboración de esta investigación.

\section{Conflictos de intereses}

Ninguno de los autores declara tener algún conflïcto de intereses.

\section{Bibliografía}

1. Bray F, Ferlay J, Soerjomataram I, Siegel RL, Torre LA, Jemal A. Global cancer statistics 2018: GLOBOCAN estimates of incidence and mortality worldwide for 36 cancers in 185 countries. CA Cancer JCClin. 2018;68:394-424.

2. Janevska D, Chaloska-Ivanova V, Janevski V. Hepatocellular carcinoma: risk factors, diagnosis and treatment. Open Access Maced J Med Sci. 2015;3:732-6

3. Jemal A, Bray F, Center MM, Ferlay J, Ward E, Forman D. Global Lcancer statistics. CA Cancer J Clin. 2011;61:69-90.

4. Ferlay J, Soerjomataram I, Dikshit R, Eser S, Mathers C, Rebelo Met al. Cancer incidence and mortality worldwide: sources, methods andmajor patterns in GLOBOCAN 2012. Int J Cancer. 2015;136:E359-86.

5. El-Serag HB, Mason AC. Rising incidence of hepatocellular carcinoma in the United States. N Engl J Med. 1999;340:745-50.

6. Ribes J, Clèries R, Borràs J, Galceran J, Bosch FX. Time trends in in incidence and mortality for chronic liver disease and liver cancer in the interval 1980-1997 in Catalonia, Spain. Eur J Gastroenterol Hepatol. 2004;16:865-72.

7. Verslype C, Rosmorduc O, Rougier P. Hepatocellular carcinoma: ESMO-ESDO Clinical Practice Guidelines for diagnosis, treatment and follow-up. Ann Oncol. 2012;23 (Suppl 7):vii41-8.

8. Cortés-Espinosa T, Mondragón-Sánchez R, Hurtado-Andrade Hánchez-Cisneros R. Hepatocellular carcinoma and hepatic cirrhosis in Mexico: a 25 year necroscopy review. Hepatogastroenterology. 1997; 44:1401-3.

9. Méndez-Sánchez N, Villa AR, Vázquez-Elizondo $\mathrm{G}$, Ponciano-Rodríguez G, Uribe M. Mortality trends for liver cancèr in Mexico from 2000 to 2006. Ann Hepatol. 2008;7:226-9.

10. Makuuchi M, Donadon M, Torzilli G. Hepatic resection for hepatocellular carcinoma in cirrhosis. Annali Italiani di Chirurgia. 2008;79:111-5.으

11. Bruix J, Sherman M, American Association for the Study of Liver Diseases. Management of hepatocellular carcinoma: an update. Hepatology. 2011;53:1020-2.

12. European Association for Study of Liver, European Organization for Research and Treatment of Cancer. EASL-EORTC Clinical Practice Guidelines: management of hepatocellular carcinoma. Eur $\mathrm{J}$ Câncer. 2012;48:599-641.

13. Mazzaferro V, Regalia E, Doci R, Andreola S, Pulvirenti A, Bozzetti F, et al. Liver transplantation for the treatment of small hepatocellular carcinomas in patients with cirrhosis. N Engl J Med. 1996;334:693-700.

14. Olthoff KM, Forner A, Hübscher S, Fung J. What is the best staging system for hepatocellular carcinoma in the setting of liver transplantation? Liver Transpl. 2011;17 (Suppl 2):S26-33.

15. Lang H, Sotiropoulos GC, Brokalaki El, Schmitz KJ, Bertona C, Meyer G, et al. Survival and recurrence rates after resection for hepatocellular carcinoma in noncirrhotic livers. J Am Coll Surg. 2007;205:27-36.

16. Setiawan VW, Hernandez BY, Lu SC, Stram DO, Wilkens LR, Marchand LL, et al. Diabetes and racial/ethnic differences in hepatocellular carcin̈oma risk: the multiethnic cohort. J Natl Cancer Inst. 2014;106:dju326

17. Tsukuma H, Hiyama T, Tanaka S, Nakao M, Yabuuchi T, Kitamura T, et al. Risk factors for hepatocellular carcinoma among patients with chronic liver disease. N Engl J Med. 1993;328:1797-801. 
18. Nagasue N, Ogawa Y, Yukaya H, Ohta N, Ito A. Serum levels of estrogens and testosterone in cirrhotic men with and without hepatocellular carcinoma. Gastroenterology. 1985;88:768-72.

19. Prates MD, Torres FO. A cancer survey in Lourenço Marques, Portuguese East Africa. J Natl Cancer Inst. 1965;35:729-57.

20. Sherman M. Hepatocellular carcinoma: epidemiology, surveillance, and diagnosis. Semin Liver Dis. 2010;30:3-16.

21. Thorgeirsson SS, Grisham JW. Molecular pathogenesis of human hepatocellular carcinoma. Nat Genet. 2002;31:339-46.

22. Mazzanti R, Messerini L, Monsacchi L, Buzzelli G, Zignego AL, Foschi M, et al. Chronic viral hepatitis induced by hepatitis $C$ but not hepatitis $B$ virus infection correlates with increased liver angiogenesis. Hepatology. 1997;25:229-34.

23. Mazzanti R, Messerini L, Comin CE, Fedeli L, Ganne-Carrie N, Beaugrand $\mathrm{M}$. Liver angiogenesis as a risk factor for hepatocellular carcinoma development in hepatitis $\mathrm{C}$ virus cirrhotic patients. World J Gastroenterol. 2007;13:5009-14

24. Ruiz-Morales JM, Dorantes-Heredia R, Chable-Montero F, Vázquez-Manjarrez S, Méndez-Sánchez N, Motola-Kuba D. Bone metastases as the initial presentation of hepatocellular carcinoma. Two case reports and a literature review. Ann Hepatol. 2014;13:838-42.

25. Furukawa $\mathrm{K}$, Shiba $\mathrm{H}$, Horiuchi $\mathrm{T}$, Shirai $\mathrm{Y}$, Haruki $\mathrm{K}$, Fujiwara $\mathrm{Y}$, et al. Survival benefit of hepatic resection for hepatocellular carcinoma beyond the Barcelona Clinic Liver Cancer classification. J Hepatobiliary Pancreat Sci. 2017;24:199-205

26. Trevisani F, Santi V, Gramenzi A, Di Nolfo MA, Del Poggio $P$, Benvegnù $L$, et al. Surveillance for early diagnosis of hepatocellular carcinoma: is it effective in intermediate/advanced cirrhosis? Am J Gastroenterol 2007;102:2448-57.

27. Singal A, Volk ML, Waljee A, Salgia R, Higgins $P$, Rogers MA, et al. Meta-analysis: surveillance with ultrasound for early-stage hepatocellular carcinoma in patients with cirrhosis. Aliment Pharmacol Therap. 2009;30:37-47.

28. Sarasin FP, Giostra E, Hadengue A. Cost-effectiveness of screening for detection of small hepatocellular carcinoma in western patients with Child-Pugh class A cirrhosis. Am J Med. 1996;101:422-34

29. Kudo M, Izumi N, Ichida T, Ku Y, Kokudo N, Sakamoto M, et al. Report of the 19th follow-up survey of primary liver cancer in Japan. Hepatol Res. 2016;46:372-90.

30. Zhong JH, You XM, Lu SD, Wang YY, Xiang BD, Ma L, et al. Historical comparison of overall survival after hepatic resection for patients with large and/or multinodular hepatocellular carcinoma. Medicine (Balt). 2015;94:e1426

31. Ikai I, Yamamoto $Y$, Yamamoto $N$, Terajima $H$, Hatano $E$, Shimahara $Y$, et al. Results of hepatic resection for hepatocellular carcinoma invading major portal and/or hepatic veins. Surg Oncol Clin N Am. 2003;12:65-75.

32. Facciuto ME, Koneru B, Rocca JP, Wolf DC, Kim-Schluger L, Visintainer $P$, et al. Surgical treatment of hepatocellular carcinoma beyond Milan criteria. Results of liver resection, salvage transplantation and primary liver transplantation. Ann Surg Oncol. 2008;15:1383-91.

33. Zhong J-H, Ke Y, Gong W-F, Xiang B-D, Ma L, Ye X-P, et al. Hepatic resection associated with good survival for selected patients with intermediate and advanced-stage hepatocellular carcinoma. Ann Surg. 2014;260:329-40.

34. Zhong JH, You XM, Lu SD, Wang YY, Xiang BD, Ma L, et al. Historical comparison of overall survival after hepatic resection for patients with large and/or multinodular hepatocellular carcinoma. Medicine (Balt). 2015;94:e1426
35. Liu W, Wang K, Bao Q, Sun Y, Xing BC. Hepatic resection provided long-term survival for patients with intermediate and advanced stage resectable hepatocellular carcinoma. World J Surg Oncol. 2016;14;62.

36. Jarnagin WR. Management of small hepatocellular carcinoma: a review of transplantation, resection, and ablation. Ann Surg Oncol. 2010;17:1226-33.

37. Hou YF, Li B, Wei YG, Yang JY, Wen TF, Xu MQ, et al. Second h్7epatectomy improves survival in patients with microvascular invasive hepatocellular carcinoma meeting the Milan criteria. Medicine (Balt). 2015;94:e2070

38. Song T. Recent advances in surgical treatment of hepatocellularearcinoma. Drug Discov Ther. 2015;9:319-30.

39. Hackl C, Schlitt HJ, Kirchner GI, Knoppke B, Loss M. Liver transptäntation for malignancy: current treatment strategies and future perspeetives. World J Gastroenterol. 2014;20:5331-44.

40. Zhou J, Hu Z, Zhang Q, Li Z, Xiang J, Yan S, et al. Spectrum of de novo cancers and predictors in liver transplantation: analysis of the Scientific Registry of Transplant Recipients Database. PloS Cone. 2016;11:e0155179.

41. Finkenstedt A, Vikoler A, Portenkirchner M, Mülleder K, Maglione M, Margreiter $\mathrm{C}$, et al. Excellent post-transplant survival in patients, with intermediate stage hepatocellular carcinoma responding to neoadjuvant therapy. Liver Int. 2016;36:688-95.

42. Sandhu L, Sandroussi C, Guba M, Selzner M, Ghanekar A, Cattrat MS, et al. Living donor liver transplantation versus deceased donopliver transplantation for hepatocellular carcinoma: comparable survivå and recurrence. Liver Transpl. 2012;18:315-22.

43. Mazzaferro V, Llovet JM, Miceli R, Bhoori S, Schiavo M, Mariani L, et al. Predicting survival after liver transplantation in patients with hepatocellular carcinoma beyond the Milan criteria: a retrospective, exploratory analysis. Lancet Oncol. 2009;10:35-43.

44. Choi HJ, Kim DG, Na GH, Han JH, Hong TH, You YK. Clinical outcome in patients with hepatocellular carcinoma after living-donor liver fransplantation. World J Gastroenterol. 2013;19:4737-44.

45. Jiang W, Li J, Guo Q, Sun J, Chen C, Shen Z. Liver transplantation for hepatocellular carcinoma. Drug Discov Ther. 2015;9:331-4

46. Pose E, Trebicka J, Mookerjee RP, Angeli P, Ginès P. Statins: olddrugs as new therapy for liver diseases? J Hepatol. 2019;70:194-202.

47. Thrift AP, Natarajan Y, Liu Y, El-Serag HB. Statin use after diagnosis of hepatocellular carcinoma is associated with decreased mortality. Clin Gastroenterol Hepatol. 2019;17:2117-25.e3.

48. Siegel RL, Miller KD, Jemal A. Cancer statistics, 2020. CA Cancer PClin. 2020;70:7-30.

49. Alqahtani A, Khan Z, Alloghbi A, Said Ahmed TS, Ashraf M, Hammouda DM. Hepatocellular carcinoma: molecular mechanisms and targeted therapies. Medicina (Kaunas). 2019;55:526.

50. Montalvo-Javé EE, Hoyos-Lazaro AE. Tumores Hepáticos. En: Academia Mexicana de Cirugía. Clínicas Quirúrgicas: Temas Selectos en Patología Hepatopancreatobiliar. Vol. XXI. Ciudad de México: Abdo-Francis JM, Pérez-Torres E. 2020. [citado 12 agosto 2020]. pp. 187-213.

51. Zamora-Valdes D, Taner T, Nagorney DM. Surgical treatment of hepatocellular carcinoma. Cancer Control. 2017;24:1073274817729258.

52. Kow AWC. Transplantation versus liver resection in patients with hepatocellular carcinoma. Transl Gastroenterol Hepatol. 2019;4:33.

53. Armengol C, Sarrias MR, Sala M. Hepatocellular carcinoma: present and future. Med Clin (Barc). 2018:150:390-7. 